\title{
Unusual formation of the musculocutaneous and median nerves: a case report refined by intraneural dissection and literature review
}

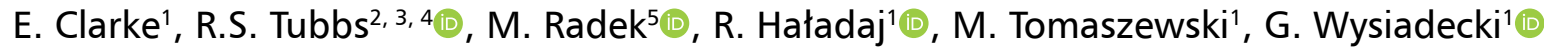 \\ ${ }^{1}$ Department of Normal and Clinical Anatomy, Chair of Anatomy and Histology, Medical University of Lodz, Poland \\ 2Department of Neurosurgery, Tulane University School of Medicine, New Orleans, LA, United States \\ ${ }^{3}$ Department of Neurosurgery and Ochsner Neuroscience Institute, Ochsner Health System, New Orleans, LA, United States \\ ${ }^{4}$ Department of Anatomical Sciences, St. George's University, Grenada \\ ${ }^{5}$ Department of Neurosurgery, Spine and Peripheral Nerve Surgery, Medical University of Lodz, University Hospital \\ WAM-CSW, Lodz, Poland
}

[Received: 20 August 2020; Accepted: 7 September 2020; Early publication date: 12 October 2020]

\begin{abstract}
This report presents a detailed anatomical investigation of an upper limb specimen showing an atypical formation of the musculocutaneous nerve (MCN) and median nerve $(M N)$. The study was refined by intraneural dissection, which supplements earlier descriptions of similar anatomical variations and allows for revision of the accepted classification.

The case described in this report was an incidental finding during routine dissection of a fixed isolated upper limb. Intraneural dissection revealed partial fusion between the MCN and aberrant bundles of the MN. Those aberrant bundles joined the main steam of the MN at the level at which the MCN branched off as an independent nerve. The procedure allowed the aberrant fibres of the $M N$ to be differentiated from the MCN. The presence of separate bundles in a territory corresponding to the MCN was confirmed, although those bundles and the aberrant MN bundles were covered by a common epineurium. The aberrant $M N$ bundles running within the MCN did not contribute to innervation of the forearm muscles. They rejoined the main nerve trunk in the arm.

A comprehensive understanding of the diverse anatomical variations of the upper limb nerves could be crucial for the safety and success of surgical procedures, especially procedures for reconstructing the brachial plexus or its branches. (Folia Morphol 2021; 80, 4: 1020-1026)
\end{abstract}

Key words: anatomical variation, brachial plexus, median nerve, musculocutaneous nerve, peripheral nerves

\section{INTRODUCTION}

The musculocutaneous nerve (MCN) is typically derived from the $\mathrm{C} 5, \mathrm{C} 6$ and $\mathrm{C} 7$ spinal cord segments. It is a direct continuation of the lateral cord of the brachial plexus. Bergman et al. [3] reported that in about $90.5 \%$ of cases the MCN shows a typical origin. During its later course it pierces the coracobrachialis muscle at the lower border of the axillary cavity. It then runs between the biceps brachii and brachialis muscles. It provides branches to both those muscles 
and travels to the lateral side of the biceps, where it pierces the brachial fascia lateral to biceps brachii muscle to emerge lateral to its distal tendon and brachioradialis muscle, and then continues as the lateral antebrachial cutaneous nerve, also known as the lateral cutaneous nerve of the forearm $[22,31]$.

In most cases (about $85 \%$ ) the median nerve (MN) arises from two roots (the terms "branches", "heads" or "radices" are occasionally used), lateral and medial, which take origin from the lateral and medial cords of the brachial plexus, respectively [22]. The lateral root provides fibres derived from ventral roots of the C5-C7 spinal nerves, while the medial cord typically contains contributions from C8 and T1. Typically, the two roots merge within the axillary cavity anterior to the axillary artery. The V-shaped origin of the MN thus created is called "the loop of the median nerve" [6]. During its further course, the MN descends the arm, accompanying the brachial artery. It seldom provides muscular branches to the arm [22]. Its territory includes the anterior group of forearm muscles and it terminates by supplying the muscles and the skin of the hand [31].

Both the MCN and MN exhibit numerous anatomical variants with respect to their origins from the brachial plexus, topographical relationships, anastomotic connections with the neighbouring nerves, and branching patterns $[3,5-8,10-23,25,27,30$, 33]. A comprehensive understanding of the diverse anatomical variations of the upper limb nerves could be crucial for the safety and success of surgical procedures, especially procedures for reconstructing the brachial plexus or its branches, particularly since the distribution of brachial plexus lesions is also highly variable $[9,11,15,18,24,29,34]$. As stressed by Sinha et al. [29], knowledge of the fascicular orientation of the nerves of the brachial plexus is important for neurosurgeons and can "improve the surgical outcome of nerve grafting in brachial plexus injuries by anastomosing related fascicles and avoiding possible axonal misrouting". In this context, refinement of gross anatomical descriptions of brachial plexus variations by intraneural dissection could help to deepen and organize existing knowledge about deviations from typical morphology and the targeting of nerves derived from that plexus. However, the technique of intraneural dissection is not commonly used.

The present report details the anatomical investigation of an upper limb specimen showing an atypical formation of the MCN and MN. The study was refined by intraneural dissection. It supplements earlier descriptions of similar anatomical variations and allows for revision of the accepted classification. One of our aims was to demonstrate the usefulness of intraneural dissection in anatomical research. Both clinical significance of the observed variation and surgical importance of the findings in this study were also discussed.

\section{CASE REPORT}

The case described in this report was an incidental finding during routine dissection of an isolated upper limb fixed in $10 \%$ formalin. Detailed examination of an atypical MCN and MN formation was supplemented by intraneural dissection using microsurgical instruments at a magnification of $2.5 \times$ under a HEINE ${ }^{\circledast}$ HR $2.5 \times$ High Resolution Binocular Loupe (HEINE Optotechnik GmbH \& Co. KG, Herrsching, Germany). The measurements were taken with a Digimatic Calliper (Mitutoyo Corporation, Kawasaki-shi, Kanagawa, Japan). Measurements were performed by two independent researchers. Each measurement was repeated twice and an average was calculated and as final result, with an accuracy of two decimal places.

In this case, the deviations from typical MCN and $\mathrm{MN}$ morphologies coincided. The variation involved partial fusion between the two nerves, which during initial assessment resembled a double loop of the MN (Fig. 1). The diameter of the lateral cord of the brachial plexus, measured at the level of the third part of the axillary artery, was $5.53 \mathrm{~mm}$; the diameter of its medial cord, measured at the same level, was $6.58 \mathrm{~mm}$. The first, typical loop of the MN covered the third part of the axillary artery (Fig. 1). The two roots of the $\mathrm{MN}$ merged just below the origin of the subscapular artery (the medial root was crossed anteriorly by this artery). The diameter of the lateral root was $3.12 \mathrm{~mm}$ and that of the medial root was $4.18 \mathrm{~mm}$. An additional band of fascicles $(1.75 \mathrm{~mm}$ in diameter), connecting the lateral cord of the brachial plexus to the medial cord of the plexus and the ulnar nerve, ran just above the two roots (Figs. 1, 2); this communication crossed the third part of the axillary artery anteriorly below the origin of the subscapular artery (Fig. 1). The MCN was remarkably thickened and resembled an elongated lateral cord of the brachial plexus (Fig. 1). Its diameter below the origin of the lateral root of the $\mathrm{MN}$ was $4.87 \mathrm{~mm}$. It ran anterior to the brachioradialis muscle (which was one-headed and not pierced by any nerve), perpendicular to the 


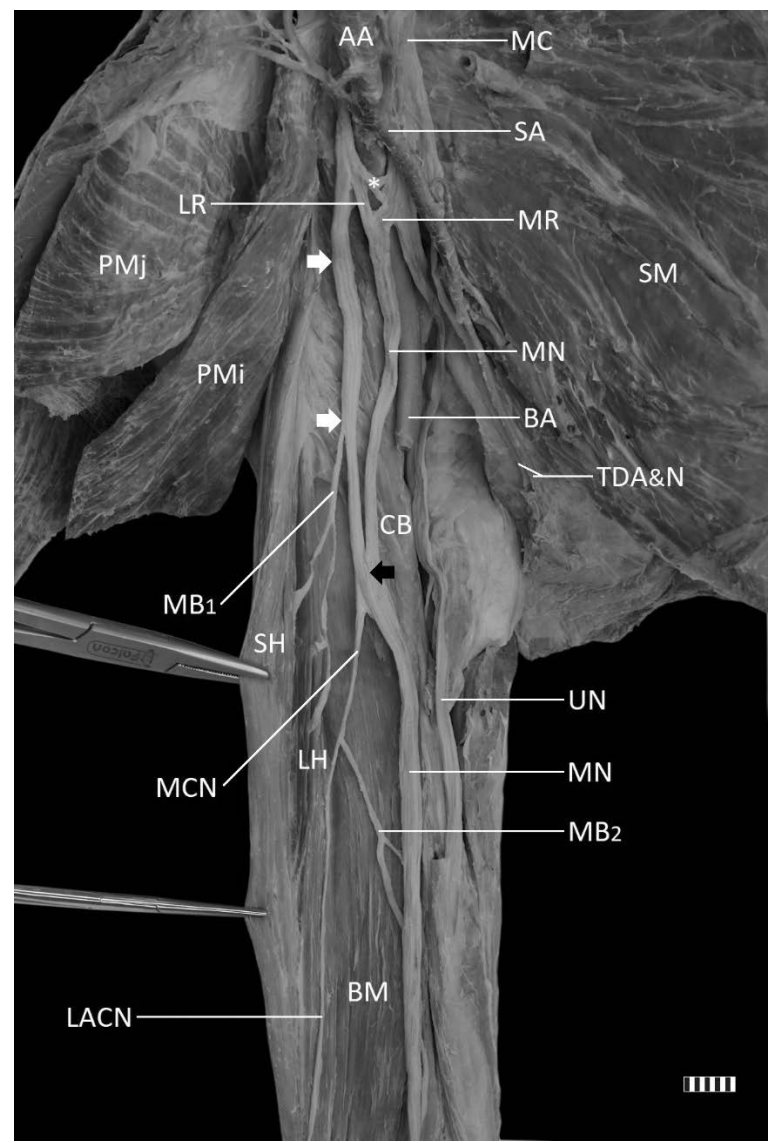

Figure 1. Co-occurrence of deviations from musculocutaneous (MCN) and median nerve (MN) typical morphology. Anterior view of the isolated right upper limb. The point at which those nerves merge is marked by a black arrow. An additional band of fascicles (marked by a white asterisk) connecting the lateral cord of the brachial plexus to the medial cord (MC) of the plexus and with the ulnar nerve (UN) runs just above the two roots of the MN. White arrows indicate an atypical thickened segment of the MCN accompanied by fibres of the MN; AA - axillary artery; BA — brachial artery (removed during dissection of the distal two-thirds of the arm); $\mathrm{BM}$ - brachialis muscle; $\mathrm{CB}$ - coracobrachialis muscle; LACN — lateral antebrachial cutaneous nerve; $\mathrm{LH}$ - long head of biceps brachii muscle; LR - lateral root of the MN; MR - medial root of the $M N$; $\mathrm{MB}_{1}$ - branch to biceps brachii muscle; $\mathrm{MB}_{2}$ branch to brachialis muscle; $\mathrm{SA}$ - subscapular artery; $\mathrm{SH}$ - short head of biceps brachii muscle; SM - subscapularis muscle;

$\mathrm{PMi}$ - pectoralis minor muscle (reflected); $\mathrm{PMj}$ — pectoralis major muscle (reflected); TDA\&N — thoracodorsal artery and nerve.

proximal third of the MN (diameter $4.31 \mathrm{~mm}$; Fig. 1). This anomalous, thickened segment of the MCN gave off one muscular branch (diameter $0.98 \mathrm{~mm}$ ) to the coracobrachialis muscle and another to the biceps brachii muscle (1.16 mm diameter; Fig. 1). Fifty-seven millimetres below the inferior border of the pectoralis major muscle, the MCN rejoined the trunk of the MN, resembling a second loop of the MN (Fig. 1). Next, the MCN (with a smaller diameter of $2.39 \mathrm{~mm}$ ) branched off again $9 \mathrm{~mm}$ below the level of its fusion

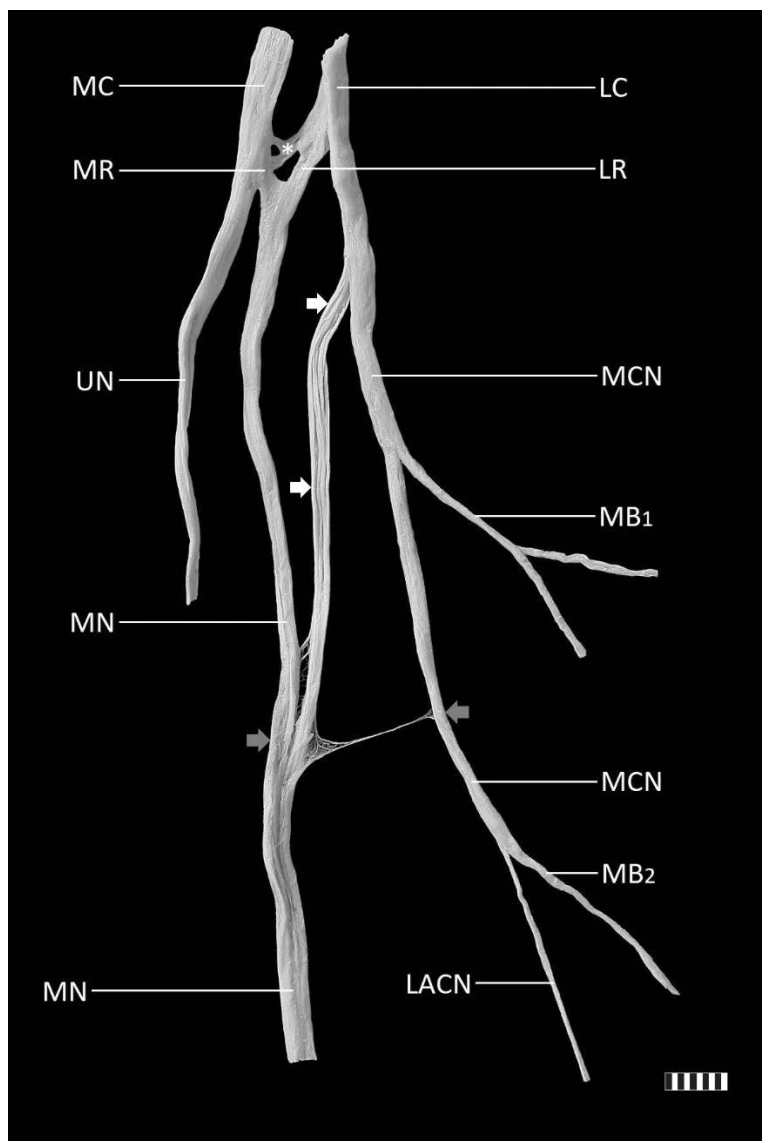

Figure 2. Result of intraneural dissection performed to revise the variation observed in Figure 1. Posterior view of the isolated specimen of part of the right brachial plexus. The fascicular dissection revealed fusion between the musculocutaneous nerve (MCN) and aberrant fibres of the median nerve (MN); those aberrant fibres are marked by white arrows. They joined the mains team of the MN at the level at which the MCN branched off as an independent nerve (marked by a grey arrow); LACN — lateral antebrachial cutaneous nerve; LC — lateral cord of the MN; LR - lateral root of the MN; $\mathrm{MC}$ - medial cords of the MN; MR - medial root of the MN; $\mathrm{MB}_{1}$ - muscular branch to the biceps brachii muscle; $\mathrm{MB}_{2}$ - branch to brachialis muscle; UN — ulnar nerve; white asterisk — additional band of fascicles connecting the lateral cord of the brachial plexus to the medial cord of the plexus and the ulnar nerve.

with the MN and $66 \mathrm{~mm}$ below the inferior border of the pectoralis major muscle. It soon entered between the biceps brachii and brachialis muscles and then followed a typical course. In the later part of its course the nerve gave a muscular branch $(0.96 \mathrm{~mm}$ diameter) to the brachialis muscle and continued as the lateral cutaneous nerve of the forearm $(1.74 \mathrm{~mm}$ diameter; Fig. 1). Just below the level of the fusion, the MN crossed the brachial artery and moved to its medial side. The subsequent course and branching pattern of the MN were typical.

The next stage of examination involved fascicular dissection to refine the classification of this varia- 
tion. The brachial plexus was harvested (including the atypical MN and MCNs) and immersed in $10 \%$ acetic acid for 2 weeks to facilitate removal of the epineural sheath according to the fascicular dissection procedure [13]. This procedure revealed the fusion between the MCN and aberrant fibres of the MN (Fig. 2); those aberrant fibres joined the main steam of the MN at the level where the MCN branched off as an independent nerve (Fig. 2). It allowed the aberrant fibres of the MN to be differentiated from the MCN. It also revealed that the muscular branch to the biceps brachii muscle came from the nerve bundle (trunk) corresponding to the MCN (Fig. 2). Aberrant fibres of the $M N$ running within the MCN did not contribute to innervation of the forearm muscles. They rejoined the main nerve trunk in the arm. The intraneural dissection results allowed the observed findings to be properly classified.

\section{DISCUSSION}

When neurovascular structures grow and differentiate within the developing limb bud, some deviations from the typical course of organogenesis are possible. These can lead to anatomical variations $[4,13,14]$. The case presented in this report describes an aberrant course of the nerve fibres derived from the brachial plexus and enables it to be analysed.

Anatomical variants can include the relationship of the first MCN segment to the coracobrachialis muscle; the MCN can lie anterior to that muscle instead of piercing it. Claassen et al. [6] estimated the incidence of this aberrant course of the nerve at $1.8 \%$. In other variants, part of the MCN pierces the coracobrachialis muscle and then rejoins the main trunk after passing through it; or only the cutaneous branch of the MCN (i.e. the lateral cutaneous nerve of the forearm) pierces that muscle $[3,22]$.

Numerous variations have been reported in the courses and anatomical relationships of the MCN and MN. However, it is challenging to compare the different classifications applied by various authors because their criteria are not uniform [27, 30]. The literature is ambiguous about the classification of variations in which there is complete or partial fusion between the MCN and MN. The MCN can be fused with the MN over a certain distance and then branch off and continue its course as a single trunk or as several branches. Such cases are sometimes classified as the MCN being a branch of the MN, and sometimes as absence of the MCN $[1,7,11,15,22,30]$.
According to Bergman et al. [3], the prevalence of the MCN branching off the $\mathrm{MN}$ ranges from $0.6 \%$ to $2 \%$. In research of Guerri-Guttenberg and Ingolotti [11], the MC was absent in 2 out of 54 cases and in 1 case the MC and the median nerve had a distal origin. Sirico et al. [30] noted in their extensive meta-analysis that fusion between the MCN and MN and the origin of the MCN from the MN can be placed in the same category, as reflected in some scientific reports. However, there are other approaches. For example, in series of Beheiry [2], the MN gave off muscular branches to the brachialis muscle as well as a branch from its lateral root to supply both heads of the biceps brachii muscle, while the MCN was reported to be absent. Thus, various cases can be classified as absence of the MCN when branches to the brachialis and biceps brachii muscles and the lateral antebrachial cutaneous nerve emerge from the MN. Our case resembles this variant, considering that an MCN following a typical course branched off only just below the place of fusion with the MN. However, in the light of the intraneural dissection results, it seems most justifiable to class this variant as "partial fusion between the MCN and MN". The presence of separate nerve bundles with territories corresponding to the MCN was confirmed, though those bundles and the aberrant MN fascicles were covered by a common epineurium. A recent report describing fascicular dissection of a case with absent MCN also revealed distinct fascicles corresponding to the MCN accompanying the MN [7]. Nerve fibres tracing of branches to the coracobrachialis muscle was also assessed by Kawashima et al. [18].

The MCN can also be accompanied by fibres derived from the MN. Such aberrant fibres can form an anastomosis between the MCN and the MN [27]. Cases of this kind could also resemble the one presented in our report. Sirico et al. [30] stress that variations in the communication between the MCN and MN may be classified as: communicating branches of the MCN; double lateral root of the MN; or "the third root of the median nerve". Communications with the MN usually appear in the lower third of the arm and can form a loop $[3,6,17,30,33]$. In research of Guerri-Guttenberg and Ingolotti [11], communications were seen between the MC and MN in 53.6\% of the dissections from which $84.6 \%$ were proximal, $7.7 \%$ distal, and $7.7 \%$ had one proximal and one distal communication to the point of entry of the MC into coracobrachialis muscle. Loukas and Aqueelah [20], 
in turn, identified four different patterns of communications between $\mathrm{MN}$ and MCN. Type I (45\%): the communications were proximal to the point of entry of the MCN into the coracobrachialis, type II (35\%): the communications were distal to the point of entry of the MCN into the coracobrachialis, type III (9\%): the MCN did not pierce the coracobrachialis and type IV (8\%): the communications were proximal to the point of entry of the MCN into the coracobrachialis and additional communication took place distally. Concomitantly the MCN was absent. Based on their own research, Maeda et al. [21] concluded, that the MCN and MN have the possibility of forming plexuses in the arm. Further studies supplemented by intraneural dissection on various cases of atypical MN and MCN formation should enable the distribution and origin of those aberrant nerve fibres to be traced. Fine dissection makes the nerve communications and separations clearly visible. For instance, studies of the MCN and MN by Kawashima et al. [18] and Maeda et al. [21] were performed with removing the epineurium under water immersion.

Bergman et al. [3] provide a concise and clear explanation of phenomena relating to atypical formations of nerves. The cited authors describe them as: "errors in the pathway (course) of some, inappropriately placed nerve fibres. For these nerve fibres to get to their proper endpoint, the bundle of nerve fibres leaves the inappropriate trunk and joins the proper nerve trunk". The background of anatomical variations among neurovascular structures based on molecular signals and factors was stressed by Carmeliet [4]. Comparative anatomical research could explain the occasional close relationship between the MN and MCN. Since the MCN is absent in lower vertebrates, the lack of a separate trunk of this nerve in humans could be considered a phylogenetic remnant [22]. Embryological studies also suggest that the MCN is derived from the MN [22]. Case of absence of the MCN associated with a supernumerary head of biceps brachii was described by Pacholczak et al. [25]. Thus, combinations of anatomical variations should be taken into account in the clinical practice $[6,7,14,19,24-28]$.

Awareness of variable anatomy of the brachial plexus and its branches could influence the safety and success of surgical procedures $[12,16,17,32]$. This is perhaps reflected in the case described by Hagemann et al. [12], who reported an adverse clinical outcome of a MN to MCN transfer abandoned because of a neuroanatomical variation. Many surgical exposures of the humerus could potentially harm the MCN $[9,26,32,35]$. An atypical MCN or an elongated lateral cord of the brachial plexus occupying an atypical position anterior to the coracobrachialis muscle seem to be at greater risk of injury during surgery [35].

Atypical formations of nerves can also influence the clinical manifestation of neurological lesions after repair of a nerve injury $[13,16,29,32]$. Sinha et al. [29] emphasized that the first factor influencing repair of a nerve lesion is the number of axons that successfully cross the anastomotic site. According to those authors, approximately $30 \%$ of axons are lost while traversing one anastomotic site. In this regard, Sinha et al. [29] conclude that "The knowledge of exact fascicular location might be translatable to the operating room and can be used to anastomose related fascicles in brachial plexus surgery, thereby avoiding the possibility of axonal misrouting and improving the results of plexal reconstruction". Considering the numerous variations of the $\mathrm{MN}$ and $\mathrm{MCN}$, advanced anatomical studies using intraneural dissection could fill the gaps in our knowledge of the origin of nerve fibres and help clinicians to deal with atypical cases [7]. It may be concluded, after Guerri-Guttenberg and Ingolotti [11], that the knowledge of these nerve's variations "will allow physicians to correctly interpret anomalous innervation patterns of the upper limb". It should also be noted that anatomical variability is the rule [36].

\section{CONCLUSIONS}

When the musculocutaneous nerve does not penetrate the coracobrachialis muscle, it may have a close relationship with the median nerve. The intraneural dissection confirmed the presence of separate fascicles corresponding to the MCN in cases of atypical formation of the MN and MCN. Awareness and detailed knowledge of anatomical variations can be crucial during clinical examination, brachial plexus repair or peripheral nerve surgeries (level of evidence IV, case series).

\section{Acknowledgements}

The authors wish to express their gratitude to all those who donated their bodies to medical science (Iwanaga J, Singh V, Ohtsuka A, et al. Acknowledging the use of human cadaveric tissues in research papers: Recommendations from anatomical journal editors. Clin Anat. 2021; 34(1): 2-4).

Conflict of interest: None declared 


\section{REFERENCES}

1. Aydin ME, Kale A, Edizer M, et al. Absence of the musculocutaneous nerve together with unusual innervation of the median nerve. Folia Morphol. 2006; 65(3): 228-231, indexed in Pubmed: 16988921.

2. Beheiry EE. Anatomical variations of the median nerve distribution and communication in the arm. Folia Morphol. 2004; 63(3): 313-318, indexed in Pubmed: 15478107.

3. Bergman RA, Afifi AK, Miyauchi R. (2015) Illustrated encyclopedia of human anatomic variation, opus III: nervous system: plexuses: musculocutaneous nerve. https://www. anatomyatlases.org/AnatomicVariants/NervousSystem/ Text/MusculocutaneousNerve.shtml.

4. Carmeliet P. Blood vessels and nerves: common signals, pathways and diseases. Nat Rev Genet. 2003; 4(9): 710-720, doi: 10.1038/nrg1158, indexed in Pubmed: 12951572.

5. Chentanez V, Jaruprat P, Udomchaisakul P, et al. Multiple variations in the course and motor branching pattern of the musculocutaneous nerve with unusual communication with the median nerve. Folia Morphol. 2016; 75(4): 555-559, doi: 10.5603/FM.a2016.0014, indexed in Pubmed: 27830893.

6. Claassen $\mathrm{H}$, Schmitt $\mathrm{O}$, Wree A, et al. Variations in brachial plexus with respect to concomitant accompanying aberrant arm arteries. Ann Anat. 2016; 208: 40-48, doi: 10.1016/j. aanat.2016.07.007, indexed in Pubmed: 27507152.

7. Clarke E, Wysiadecki G, Haładaj R, et al. Fusion between the median and musculocutaneous nerve: A case study. Folia Med Cracov. 2019; 59(3): 45-52, indexed in Pubmed: 31891359.

8. Darvishi M, Moayeri A. Anatomical variations of the musculocutaneous and median nerves: a case report. Folia Med (Plovdiv). 2019; 61(2): 327-331, doi: 10.2478/ folmed-2018-0080, indexed in Pubmed: 31301650.

9. Das KK, Joseph J, Gosal JS, et al. "Undercutting of the corresponding rib": a novel technique of increasing the length of donor in intercostal to musculocutaneous nerve transfer in brachial plexus injury. Br J Neurosurg. 2019 [Epub ahead of print]: 1-4, doi: 10.1080/02688697.2019.1630554, indexed in Pubmed: 31208238.

10. Gelmi C, Pedrini F, Fermi M, et al. Communication between median and musculocutaneous nerve at the level of cubital fossa: a case report. Transl Res Anat. 2018; 11: 1-4, doi: 10.1016/j.tria.2018.04.001.

11. Guerri-Guttenberg RA, Ingolotti M. Classifying musculocutaneous nerve variations. Clin Anat. 2009; 22(6): 671-683, doi: 10.1002/ca.20828, indexed in Pubmed: 19637305.

12. Hagemann C, Stücker R, Breyer $S$, et al. Nerve transfer from the median to musculocutaneous nerve to induce active elbow flexion in selected cases of arthrogryposis multiplex congenita. Microsurgery. 2019; 39(8): 710-714, doi: 10.1002/micr.30451, indexed in Pubmed: 30891796.

13. Haładaj R, Wysiadecki G, Macchi V, et al. Anatomic variations of the lateral femoral cutaneous nerve: remnants of atypical nerve growth pathways revisited by intraneural fascicular dissection and a proposed classification. World Neurosurg. 2018; 118: e687-e698, doi: 10.1016/j. wneu.2018.07.021, indexed in Pubmed: 30010076.

14. Haładaj R, Wysiadecki G, Polguj M, et al. Hypoplastic superficial brachioradial artery coexisting with atypical formation of the median and musculocutaneous nerves: a rare combination of unusual topographical relationships. Surg Radiol Anat. 2019; 41(4): 441-446, doi: 10.1007/ s00276-019-02183-1, indexed in Pubmed: 30652211.

15. Hayashi M, Shionoya K, Hayashi S, et al. A novel classification of musculocutaneous nerve variations: The relationship between the communicating branch and transposed innervation of the brachial flexors to the median nerve. Ann Anat. 2017; 209: 45-50, doi: 10.1016/j. aanat.2016.08.004, indexed in Pubmed: 27765675.

16. Isaacs J, Ugwu-Oju O. High median nerve injuries. Hand Clin. 2016; 32(3): 339-348, doi: 10.1016/j. hcl.2016.03.004, indexed in Pubmed: 27387077.

17. Kara $A B$, Elvan Ö, Öztürk NC, et al. Communications of the median nerve in foetuses. Folia Morphol. 2018; 77(3): 441-446, doi: 10.5603/FM.a2017.0107, indexed in Pubmed: 29131277.

18. Kawashima T, Yoshitomi S, Sasaki H. Nerve fibre tracing of branches to the coracobrachialis muscle in a Bornean orangutan (Pongo pygmaeus pygmaeus). Anat Histol Embryol. 2007; 36(1): 19-23, doi: 10.1111/j.14390264.2006.00714.x, indexed in Pubmed: 17266662.

19. Kimura S, Amatani $\mathrm{H}$, Nakai $\mathrm{H}$, et al. A novel case of multiple variations in the brachial plexus with the middle trunk originating from the $C 7$ and C8. Anat Sci Int. 2020; 95(4): 559-563, doi: 10.1007/s12565-020-00541-3, indexed in Pubmed: 32333262.

20. Loukas $\mathrm{M}$, Aqueelah $\mathrm{H}$. Musculocutaneous and median nerve connections within, proximal and distal to the coracobrachialis muscle. Folia Morphol. 2005; 64(2): 101-108, indexed in Pubmed: 16121328.

21. Maeda S, Kawai K, Koizumi M, et al. Morphological study of the communication between the musculocutaneous and median nerves. Anat Sci Int. 2009; 84(1-2): 34-40, doi: 10.1007/s12565-008-0005-y.

22. Mahan MA, Spinner RJ. Nerves of the upper extremity. In: Tubbs RS, Shoja MM, Loukas M. Bergman's comprehensive encyclopedia of human anatomic variation. Wiley, Hoboken 2016: 1068-1112.

23. Nakatani T, Tanaka S, Mizukami S. Absence of the musculocutaneous nerve with innervation of coracobrachialis, biceps brachii, brachialis and the lateral border of the forearm by branches from the lateral cord of the brachial plexus. J Anat. 1997; 191 ( Pt 3): 459-460, doi: 10.1046/j.14697580.1997.19130459.x, indexed in Pubmed: 9419004.

24. Olewnik $\measuredangle$, Paulsen F, Tubbs RS, et al. Potential compression of the musculocutaneous, median and ulnar nerves by a very rare variant of the coracobrachialis longus muscle. Folia Morphol. 2021; 80(3): 707-713, doi: 10.5603/ FM.a2020.0085, indexed in Pubmed: 32844391.

25. Pacholczak R, Klimek-Piotrowska W, Walocha JA. Absence of the musculocutaneous nerve associated with a supernumerary head of biceps brachii: a case report. Surg Radiol Anat. 2011; 33(6): 551-554, doi: 10.1007/s00276-0100771-9, indexed in Pubmed: 21225427.

26. Pećina $M$, Bojanić $I$. Musculocutaneous nerve entrapment in the upper arm. Int Orthop. 1993; 17(4): 232-234, doi: 10.1007/BF00194185, indexed in Pubmed: 8407039.

27. Roy J, Henry BM, Pekala PA, et al. Median and ulnar nerve anastomoses in the upper limb: A meta-analysis. Muscle 
Nerve. 2016; 54(1): 36-47, doi: 10.1002/mus.24993, indexed in Pubmed: 26599506.

28. Schwerdtfeger $L$, Pascoe M, Clapp T. High incidence of a third head of biceps brachii in females. Transl Res Anat. 2018; 12: 25-27, doi: 10.1016/j.tria.2018.08.001.

29. Sinha S, Prasad GL, Lalwani S. A cadaveric microanatomical study of the fascicular topography of the brachial plexus. J Neurosurg. 2016; 125(2): 355-362, doi: 10.3171/2015.6.JNS142181, indexed in Pubmed: 26654179.

30. Sirico F, Castaldo C, Baioccato V, et al. Prevalence of musculocutaneous nerve variations: Systematic review and meta-analysis. Clin Anat. 2019; 32(2): 183-195, doi: 10.1002/ca.23256, indexed in Pubmed: 30113088.

31. Standring S. Gray's anatomy: the anatomical basis of clinical practice. Churchill Livingstone 2016: 831-832.

32 . Sunderland $\mathrm{S}$. The anatomy and physiology of nerve injury. Muscle Nerve. 1990; 13(9): 771-784, doi: 10.1002/ mus.880130903, indexed in Pubmed: 2233864.
33. Mat Taib CN, Hassan SNA, Esa N, et al. Anatomical variations of median nerve formation, distribution and possible communication with other nerves in preserved human cadavers. Folia Morphol. 2017; 76(1): 38-43, doi: 10.5603/FM.a2016.0045, indexed in Pubmed: 27665953.

34. Zapałowicz K, Radek M. The distribution of brachial plexus lesions after experimental traction: a cadaveric study. J Neurosurg Spine. 2018; 29(6): 704-710, doi: 10.3171/2018.5.SPINE171148, indexed in Pubmed: 30265223

35. Zlotolow DA, Catalano LW, Barron OA, et al. Surgical exposures of the humerus. J Am Acad Orthop Surg. 2006; 14(13): 754-765, doi: 10.5435/00124635-20061200000007, indexed in Pubmed: 17148623.

36. Żytkowski A, Tubbs R, Iwanaga J, et al. Anatomical normality and variability: Historical perspective and methodological considerations. Trans Res Anat. 2021; 23: 100105 , doi: 10.1016/j.tria.2020.100105. 